\title{
The importance of mammillary body efferents for recency memory: towards a better understanding of diencephalic amnesia
}

\author{
Andrew J. D. Nelson ${ }^{1} \cdot$ Seralynne D. Vann ${ }^{1}$
}

Received: 30 October 2015 / Accepted: 19 October 2016/Published online: 25 October 2016

(c) The Author(s) 2016. This article is published with open access at Springerlink.com

\begin{abstract}
Despite being historically one of the first brain regions linked to memory loss, there remains controversy over the core features of diencephalic amnesia as well as the critical site for amnesia to occur. The mammillary bodies and thalamus appear to be the primary locus of pathology in the cases of diencephalic amnesia, but the picture is complicated by the lack of patients with circumscribed damage. Impaired temporal memory is a consistent neuropsychological finding in Korsakoff syndrome patients, but again, it is unclear whether this deficit is attributable to pathology within the diencephalon or concomitant frontal lobe dysfunction. To address these issues, we used an animal model of diencephalic amnesia and examined the effect of mammillothalamic tract lesions on tests of recency memory. The mammillothalamic tract lesions severely disrupted recency judgements involving multiple items but left intact both recency and familiarity judgements for single items. Subsequently, we used disconnection procedures to assess whether this deficit reflects the indirect involvement of the prefrontal cortex. Crossedlesion rats, with unilateral lesions of the mammillothalamic tract and medial prefrontal cortex in contralateral hemispheres, were unimpaired on the same recency tests. These results provide the first evidence for the selective importance of mammillary body efferents for recency memory. Moreover, this contribution to recency memory is independent of the prefrontal cortex. More broadly, these findings identify how specific diencephalic structures are vital for key elements of event memory.
\end{abstract}

Andrew J. D. Nelson

NelsonA5@cardiff.ac.uk

1 School of Psychology, Cardiff University, 70 Park Place, Cardiff CF10 3AT, UK
Keywords Anterior thalamic nuclei - Diencephalic amnesia - Mammillothalamic tract - Prefrontal cortex . Rats $\cdot$ Recognition memory

\section{Introduction}

The medial diencephalon was probably the first brain region to be linked to memory loss (Gudden 1896), but there still remains much uncertainty over the core features of diencephalic amnesia and how structures within the medial diencephalon support mnemonic functions. Evidence from Korsakoff's syndrome and lacunar infarct patients indicates that the mammillary bodies and thalamus are the primary locus of pathology in diencephalic amnesia (Carlesimo et al. 2011; Harding et al. 2000; Mayes et al. 1988; Pitel et al. 2012; Van der Werf et al. 2003). However, the pathology is rarely circumscribed and, particularly in the case of Korsakoff's syndrome, there are often concomitant changes to both white matter tracts and grey matter structures beyond the medial diencephalon, including frontal lobe dysfunction (Harper and Corbett 1990; Harper 2009; Langlais et al. 1996; Torvik et al. 1982). Consequently, it has proved difficult to attribute specific cognitive impairments to particular brain regions (Kopelman 2015).

Diencephalic amnesic patients are impaired on tests of recency memory that require individuals to make judgments about the temporal context in which an item was encountered (Hildebrandt et al. 2001; Huppert and Piercy 1976; Kopelman et al. 1997; Meudell et al. 1985; Parkin et al. 1990). Temporal or recency memory is classically associated with the frontal cortex; both patients (McAndrews and Milner 1991; Milner et al. 1991; Shimamura et al. 1990) and animals (Barker et al. 2007; Petrides 1991) 
with damage to frontal regions are impaired on tasks that tax this aspect of memory. There are various possible explanations of impaired recency memory in diencephalic amnesia: it may be due to co-occurring frontal lobe dysfunction (Mayes et al. 1985; Shimamura et al. 1990; Squire 1982), it could reflect the disconnection between frontal cortex and frontal cortex-associated thalamic nuclei, such as the mediodorsal thalamus (Cross et al. 2012; Schnider et al. 1996), or alternatively, it might be a core feature of diencephalic amnesia (Hunkin and Parkin 1993; Kopelman 1989; Kopelman et al. 1997). Given the lack of patients with circumscribed diencephalic pathology, less equivocal evidence can only be obtained from animal models involving restricted damage within discrete regions of the medial diencephalon. In this respect, targeting the mammillothalamic tract (MTT) is particularly appealing, because all neurons in the mammillary bodies are thought to project to the anterior thalamus via this tract (Vann et al. 2007). MTT transection, therefore, allows a direct assessment of mammillary body contributions to medial diencephalic function.

To address these issues, we first tested rats with discrete lesions to the MTT on a variant of the recency tasks used with Korsakoff patients (Hunkin et al. 2015). This task makes use of rats' inherent preference for relative novelty (Hannesson et al. 2004), to discriminate between multiple objects presented at different time points and makes it possible to test both "within-list" and "between-list" memory. Performance on this task (between-block recency) is sensitive to both hippocampal and anterior thalamic damage (Albasser et al. 2012; Dumont and Aggleton 2013). Control experiments assessed both recency and familiarity judgements for single items. Second, we examined whether any impairments seen in MTT lesion rats could reflect frontal involvement. Bilateral medial prefrontal damage is known to disrupt simple between-block recency discriminations (Barker et al. 2007; Hannesson et al. 2004; Nelson et al. 2011). The medial mammillary bodies are directly innervated by medial prefrontal cortex (Allen and Hopkins 1989). The medial mammillary bodies can also indirectly influence the frontal cortex via their connections with the anteromedial thalamic nucleus (Hayakawa and Zyo 1989; Wright et al. 2013). In turn, the anteromedial thalamic nucleus projects unilaterally to the medial prefrontal cortex (de Lima et al. 2016; Hoover and Vertes 2007; van Groen et al. 1999). Moreover, MTT lesions in rats can disrupt markers of neuronal activity (as measured by the immediate-early gene $c$-fos) in prelimbic cortex (Vann and Albasser 2009; Vann 2013). As such, any effects of bilateral MTT lesions on recency discriminations could, in part, be mediated by disruption to information flow within prefrontal-mammillary body-anteromedial thalamic-prefrontal pathways or by distal effects of MTT lesions on prefrontal functioning. Disconnection procedures can be used to rule out these explanations. Accordingly, we tested crossed-lesion rats, with unilateral lesions of the MTT and medial prefrontal cortex in contralateral hemispheres, on the same recency memory task.

\section{Materials and methods}

\section{Subjects and surgery}

In Experiment 1, subjects were 28 male Lister Hooded rats (Harlan, Bicester, UK) weighing between 234 and $303 \mathrm{~g}$ at the time of surgery. Experiment 2 involved an additional 28 male Lister Hooded rats (Harlan, Bicester, UK) weighing between 276 and $384 \mathrm{~g}$ at the time of surgery. Animals were housed in pairs under diurnal light conditions (14 h light $/ 10 \mathrm{~h}$ dark) and testing was carried out during the light phase. Animals were given free access to water and a large cardboard tube and wooden chew-stick were available in the home-cage throughout. All experiments were carried out in accordance with UK Animals (Scientific Procedures) Act, 1986 and EU directive 2010/63/EU.

Surgery was performed under an isoflurane-oxygen mixture (2-2.5\% isoflurane). Once anaesthetised, the animals were placed in a stereotaxic head holder (David Kopf Instruments, Tujunga, CA), with the nose-bar at -3.3 (flat skull), and a longitudinal incision was made in the scalp, which was retracted to expose the skull. The skull was drilled at the point of the lesion. In Experiment 1, rats received bilateral mammillothalamic tract lesions (MTTx; $n=15$ ) made by radiofrequency using a thermocouple radiofrequency electrode $(0.7 \mathrm{~mm}$ active tip length, $0.25 \mathrm{~mm}$ diameter; Diros Technology Inc., Toronto, Canada). The electrode was lowered vertically and the tip temperature raised to $70{ }^{\circ} \mathrm{C}$ for $33 \mathrm{~s}$ using an OWL Universal RF System URF-3AP lesion maker (Diros Technology Inc., Toronto, Canada). The stereotaxic coordinates for the lesions were: anterior-posterior (AP), -2.5 ; medio-lateral (ML), \pm 0.9 (both relative to bregma); and the depth (DV), from top of cortex, was $-6.9 \mathrm{~mm}$. The surgical control rats (Sham; $n=13$ ) underwent the same procedures except the probe was lowered to $+1.0 \mathrm{~mm}$ above the lesion site and the temperature of the probe was not raised.

In Experiment 2, 16 rats received crossed mammillothalamic tract-medial prefrontal cortex lesions (i.e., a unilateral mammillothalamic tract (MTT) lesion in one hemisphere and a medial prefrontal cortex (mPFC) lesion in the contralateral hemisphere). Half of the animals received an MTT lesion in the left hemisphere and an 
mPFC lesion in the right hemisphere, while for the remaining eight animals, this order was reversed. The intended locus of the unilateral mPFC lesion was selected on the basis of the known outputs from the mPFC to the mammillary bodies and inputs to the mPFC from the anteromedial thalamic nucleus (Allen and Hopkins 1989; Hoover and Vertes 2007; van Groen et al. 1999) as well as evidence from the previous lesion studies implicating the $\mathrm{mPFC}$ in recency memory (Barker et al. 2007; Devito and Eichenbaum 2011; Hannesson et al. 2004; Hasselmo and Eichenbaum 2005; Nelson et al. 2011). The MTT lesions were conducted in the same manner as for Experiment 1 except that the lesion was only made in one hemisphere and the tip temperature of the probe was raised to $70{ }^{\circ} \mathrm{C}$ for 35 s. Following a craniotomy at the point of the lesion site, the unilateral mPFC lesions were made by injecting three sites with $0.28 \mu \mathrm{l}$ of $0.09 \mathrm{M} N$-methyl-D-aspartic acid (NMDA; Sigma). Infusions were made with a $1 \mu \mathrm{l}$ Hamilton syringe (Bonaduz, Switzerland) at an infusion rate of $0.1 \mu \mathrm{l} / \mathrm{min}$. The $\mathrm{AP}$ and $\mathrm{ML}$ co-ordinates were measured (in $\mathrm{mm}$ ) with respect to bregma, and the DV coordinates (in $\mathrm{mm}$ ) with respect to the surface of the cortex. The stereotaxic co-ordinates of the three injection sites were as follows: (1) $\mathrm{AP}=+3.8 ; \quad \mathrm{ML}= \pm 0.7$; $\mathrm{DV}=-3.8 ;$ (2) $\mathrm{AP}=+3.2 ; \mathrm{ML}= \pm 0.7 ; \mathrm{DV}=-3.6$; and (3) 3. $\mathrm{AP}=+2.5 ; \mathrm{ML}= \pm 0.7 ; \mathrm{DV}=-3.4$. The needle was left in situ for $5 \mathrm{~min}$ after each infusion. The 12 surgical controls underwent a unilateral "sham" MTT surgery as described for Experiment 1 and a "sham" unilateral mPFC lesion which was conducted in the same manner as the lesion except that no infusions of NDMA were made.

On completion of surgery, the skin was sutured and antibiotic powder (Clindamycin Hydrochloride, Pharmacia, Sandwich, UK) was applied topically to the wound-site. Animals also received subcutaneous injections of $5 \mathrm{ml}$ glucose saline and Metacam $(0.06 \mathrm{ml}$, s.c.; $5 \mathrm{mg} / \mathrm{ml}$ Meloxicam, Boehringer Ingelheim, Rhein, Germany) provided post-operative analgesia. All animals recovered well following surgery.

After a minimum of 2 -week post-operative recovery, rats were placed on a food restricted diet where they were still able to gain weight; their weights did not fall below $85 \%$ of their equivalent free feeding weight.

\section{Behavioural testing}

\section{Experiment 19 and 2: multi-item recency judgments}

These experiments made use of rats' spontaneous preference for less recently presented (i.e., more novel) objects as a measure for recency memory and involved both betweenand within-list discriminations (Dumont and Aggleton
2013). Rats were required to discriminate, on the basis of relative recency, between pairs of objects that had either been presented in separate temporal blocks (betweenblock) or within the same continuous block of trials but at different time points (within-block).

Rats underwent each recency test (between- and withinblock recency) twice. The order of testing was counterbalanced across animals, such that half of the animals were tested first on between-block recency, then on within-block recency followed by the second test of between-block recency and finally the second within-block recency test. For the other half of the animals, this order was reversed. At least 7 days separated each test.

Apparatus Testing occurred in a maze with the shape of a bow tie (120 cm long, $50 \mathrm{~cm}$ wide, and $50 \mathrm{~cm}$ high) made of aluminium (Fig. 1; Albasser et al. 2010). Each end of the maze consisted of a triangular area, and these areas were joined together at their apices by a corridor $(12 \mathrm{~cm}$ wide). In the centre of the corridor, an opaque sliding door could be lowered or raised by the experimenter to allow passage from one end of the maze to the other. At the far wall of each of the triangles, there were two food wells
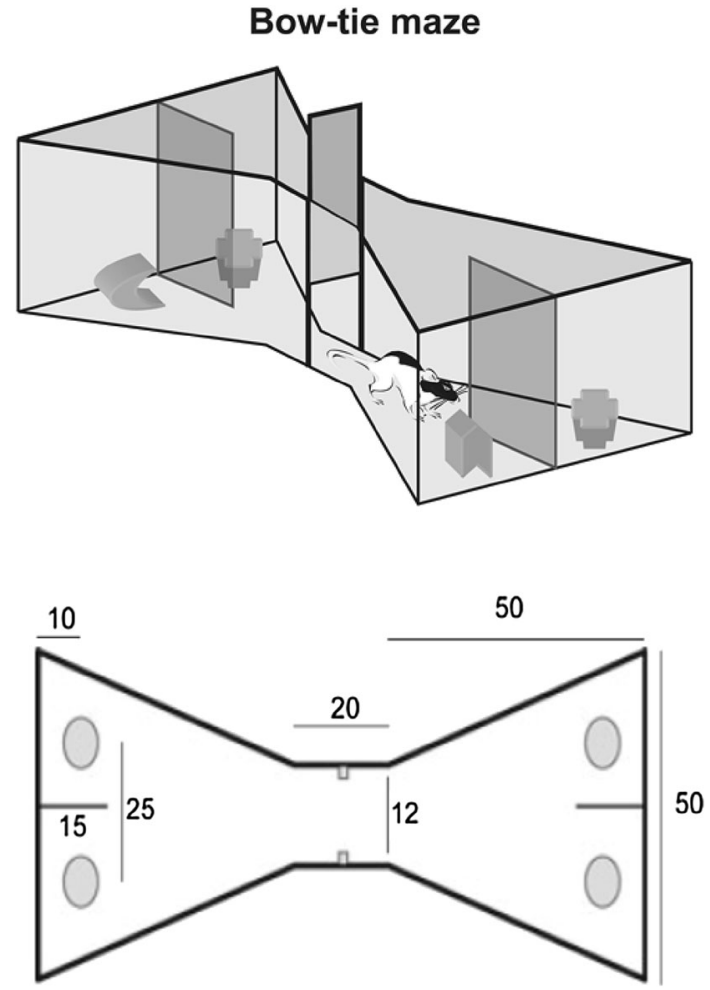

Fig. 1 Upper panel is a graphic of the test apparatus used for testing object recognition and object recency memory. A sliding door in the centre divides the maze into two halves, so that objects can be placed over the food wells in one half, while the animal is completing the task in the other half. Lower panel is a schematic of the bow-tie maze, with dimensions in centimetres 
(3.5 $\mathrm{cm}$ in diameter and $2 \mathrm{~cm}$ deep), separated by a short, opaque, wall extending $15 \mathrm{~cm}$ from the middle of the end wall. The two food wells were $25 \mathrm{~cm}$ apart. Objects were placed above these two food wells during the experiment.

Triplicate sets of identical objects that differed in size, shape, colour, and texture were used. A mixture of plastic, glass, ceramic, and wooden objects was used. Objects had to be large enough to cover one food well but also light enough for the rats to displace. The height of the objects ranged between 2 and $15 \mathrm{~cm}$ and the width ranged between 4 and $10 \mathrm{~cm}$. The presentation of objects was counterbalanced, so that half the rats experienced the list of objects in one order (e.g., A-K), whereas the other half of rats experienced the list in the reverse order (e.g., K-A). The positioning of the objects within the maze (over either left or right food well) was also counterbalanced. Different sets of objects were used for each test, so that each test contained unique items.

Habituation and pre-training Habituation lasted 7 days during which time the rats learnt to run from one end of the maze to the other and displace objects covering the food wells to obtain a sucrose reward pellet. Initially (day 1), pairs of rats were allowed to explore the maze for $20 \mathrm{~min}$ and collect sucrose pellets that had been scattered across the floor and food wells. One the next day, individual rats were trained $(10 \mathrm{~min})$ to run back and forth for rewards that were now located in the food wells. On day 3, the sliding door that restricted movement from one compartment to the other was introduced. On day 4, the rats learnt to push objects to obtain the sucrose pellets by placing four identical wooden blocks that partially, and subsequently, fully occluded the food wells. For the remaining three sessions, different pairs of objects were introduced. None of the objects used during pre-training was subsequently used during testing.

Between-block recency Each session consisted of three phases: two sample phases followed by a test phase (see Fig. 2a). Thus, rats were presented with lists of objects in two distinct, temporal blocks; at test, rats were required to discriminate between objects that had been presented in different temporal blocks. Each sample phase involved multiple trials of standard object recognition (Fig. 2a).

A

Mutliple Item Between-block Recency

\begin{tabular}{|c|c|c|c|c|c|c|c|c|c|c|c|c|c|c|c|c|c|c|c|c|c|c|c|c|c|c|c|}
\hline \multirow[b]{2}{*}{0} & \multicolumn{10}{|c|}{ Sample List 1} & \multicolumn{9}{|c|}{ Sample List 2} & \multicolumn{8}{|c|}{ Recency Test (List 1 vs. List 2) } \\
\hline & 1 & 2 & 3 & 4 & 5 & 6 & 7 & 8 & & 0 & 9 & 10 & 11 & 12 & 13 & 14 & 15 & 16 & & 17 & 18 & 19 & 20 & 21 & 22 & 22 & 24 \\
\hline- & A & B & C & D & $E$ & $\mathrm{~F}$ & $\mathrm{G}$ & $\mathrm{H}$ &. $\bar{\varepsilon}$ & - & $\mathrm{J}$ & K & $\mathrm{L}$ & $\mathrm{M}$ & $\mathrm{N}$ & 0 & $\mathrm{P}$ & Q & . & $\mathrm{J}$ & $\mathrm{K}$ & $\mathrm{L}$ & M & $\mathrm{N}$ & 0 & $P$ & $Q$ \\
\hline A & B & C & D & E & $\mathbf{F}$ & G & H & I & চ & J & K & $\mathbf{L}$ & M & $\mathbf{N}$ & 0 & $\mathbf{P}$ & $\mathbf{Q}$ & $\mathbf{R}$ & 오 & A & B & C & D & E & $\mathbf{F}$ & G & H \\
\hline
\end{tabular}

B

Multiple Item Within-block Recency

\begin{tabular}{cccccccccccccccccc|cccccccc}
\hline \multicolumn{10}{c}{ Sample Phase } & \multicolumn{1}{c}{ Recency Test Phase } \\
\hline 1 & 2 & 3 & 4 & 5 & 6 & 7 & 8 & 9 & 10 & 11 & 12 & 13 & 14 & 15 & 16 & 17 & 18 & 19 & 20 & 21 & 22 & 23 & 24 & 25 & 26 \\
\hline A & B & C & D & E & F & G & H & I & J & K & L & M & N & O & P & Q & R & M & R & K & P & Q & L & O & N \\
A & B & C & D & E & F & G & H & I & J & K & L & M & N & O & P & Q & R & E & B & G & D & A & H & C & F
\end{tabular}

C Simple Between-block Recency

\begin{tabular}{|c|c|c|c|c|c|c|c|}
\hline \multicolumn{2}{|c|}{ Sample 1} & \multirow[b]{2}{*}{$\frac{\varrho}{\mathrm{E}}$} & \multicolumn{2}{|c|}{ Sample 2} & \multirow[b]{2}{*}{$\cdot \frac{\subseteq}{E}$} & \multicolumn{2}{|c|}{ Recency Test } \\
\hline$A$ & $A$ & & $B$ & $B$ & & A & A \\
\hline A & A & ผ & B & B & 㞫 & B & B \\
\hline
\end{tabular}

\section{Standard Object Recognition}

\begin{tabular}{|c|c|c|c|c|}
\hline \multicolumn{2}{|c|}{ Sample } & \multirow[b]{2}{*}{.气 } & \multicolumn{2}{|c|}{ Test } \\
\hline$A$ & $A$ & & $A$ & $A$ \\
\hline$A$ & $A$ & 8 & B & B \\
\hline
\end{tabular}

Fig. 2 Order of object presentation in the multi-item between-block (a) and within-block (b) recency tests. Order of object presentation in the simple between-block (c) recency test and standard object recognition test (d). Items in bold refer to either novel or least recently explored objects, i.e., objects for which rats should show a preference 
During Sample 1, rats received eight trials of standard object recognition (plus a trial " 0 " in which the first object was encountered). At the beginning of the session, the rat was placed in one end of the maze that contained an object (Object A1) covering one food well and a wood block covering the other well. The rat was allowed to retrieve the food rewards and explore both objects in a trial lasting $1 \mathrm{~min}$. The sliding door was then raised allowing access to the second compartment. Once the rat ran to the opposite side of the maze the sliding door was lowered, the rat could now explore a novel item (Object B1) and a familiar item (Object A2, a duplicate of Object A1 from Trial 0). Both the novel and familiar objects covered wells that contained a reward, so every object on every trial was rewarded. After a minute, the sliding door was raised again, and the rat ran back to the first compartment of the maze (Trial 2) where Object $\mathrm{C}(\mathrm{C} 1$; novel) and a duplicate of Object B (B2; familiar) were presented. After $1 \mathrm{~min}$, the sliding door was raised again (Trial 3), and the rat ran back into the second compartment to explore a copy of Object C (C2; familiar) and new Object D (D1; novel). This process continued with different objects until 8 trials had been completed, i.e., objects A-I. Sample 2 followed after a 30 min delay and consisted of eight more trials of standard object recognition involving the new objects J-R (see Fig. 2a).

After a further 10 min delay, recency judgements were assessed by presenting rats in each trial with pairs of objects with one object from Sample 1 (less recent) and one from Sample 2 (more recent). The rat was returned to one end of the maze and copies of objects from each of the sample phases (e.g., Object A3 and Object J3) were presented. The rat had $1 \mathrm{~min}$ to explore the objects and retrieve the pellets from under both objects. The sliding door was then raised, and the rat ran to the second compartment where further copies of objects from each sample phase (e.g., B2 and K3) were presented. This procedure continued until all eight trials had been completed.

Within-block recency Each session involved an 18-trial sample phase and an 8-trial recency test phase both of which occurred within a continuous block of trials.

The sample phase began when the rat was placed in one end of the maze. The rat was allowed $1 \mathrm{~min}$ to push aside and explore two identical objects (A1, A2) that each covered a food well (Fig. 2b). The sliding door was then opened, allowing the rat to run across to the second compartment where two copies of a novel object were present (B1, B2). The rat again had $1 \mathrm{~min}$ to explore these objects and obtain the sucrose pellets. Once this trial was completed, the sliding door was again opened and the rat ran back to the first compartment where two copies of novel another novel object $(\mathrm{C} 1, \mathrm{C} 2)$ were presented. This process continued until the rats had encountered all 18 pairs of objects (i.e., A-R). Following the trial 18, the sliding door was raised allowing the rat to change compartments. The recency phase began immediately, so the rat was not removed from the apparatus or was the rat handled between two phases.

For each trial of the test phase, the rat could explore two objects that had been presented at different time points in the sample phase. As before, every object covered a food reward. For example, Trial 1 of the recency (test) phase consisted of copies of object E (E3) and object M (M3). After a minute, the rat was allowed to run to the other side of the maze to find copies of object B (B3) and object R (R3). The number of interleaving items between the two objects was set at $3,7,11$, or 15 . Trials with different numbers of interleaving items were intermixed.

\section{Experiment 1b: single-item recency judgments}

To reduce the proactive interference that arises from being presented with multiple different objects within a relatively short timeframe, this experiment assessed rats' ability to discriminate between single items that had been presented in separate temporal blocks. Each rat was tested twice, with a minimum 7-day interval between each test. Different sets of objects were used in each test, and none of the objects had been encountered previously.

Apparatus Testing occurred in the same bow-tie maze as described previously. Two sets of four identical objects were used. At test, two duplicates from each set were used. As previously, junk objects that differed in size, shape, colour, and texture were used.

Procedure Each session consisted of three phases: two sample phases followed by a test phase (see Fig. 2c). This experiment did not involve multiple continuous trials, and consequently, no reward pellets were placed under the objects, so that the animals could not displace them, the objects were larger than those in Experiment $1 \mathrm{a} / \mathrm{b}$. The order of presentation, i.e., whether the object was presented in Sample 1 or 2 was counterbalanced across animals. In Sample 1, rats were placed in the arena and were able to move freely around the entire maze and to explore four identical objects. After 5-min exploration time, the rats were removed from the arena and were returned to a holding room for $25 \mathrm{~min}$. After this delay, the rats were returned to the maze and could explore a new set of four identical objects for $5 \mathrm{~min}$. Following a further $25 \mathrm{~min}$ delay, the test phase occurred during which rats were able to explore two pairs of objects (one pair from each sample phase-see Fig. 2c). Each end of the maze contained one replica object from each sample phase. The test phase lasted $3 \mathrm{~min}$. 


\section{Experiment 1c: standard object recognition}

This experiment examined standard object recognition memory, i.e., the ability to discriminate between items on the basis of relative familiarity. MTT lesions do not disrupt this ability with relatively short retention delays, e.g., 10 min (Nelson and Vann 2014). To match the maximum delay between object presentation in the recency tests (Experiments 1a/b), this experiment examined animals' ability to discriminate a novel from a familiar object after a 60 min delay.

Apparatus Testing occurred in the same bow-tie maze as described previously. Two sets of four identical objects were used. At test, two duplicates from each set were used. As previously, junk objects that differed in size, shape, colour, and texture were used.

Procedure Each session consisted of two phases: a sample phase followed by a test phase (see Fig. 2d). This experiment did not involve multiple continuous trials, and consequently, no reward pellets were placed under the objects. The set of objects that served as familiar or novel was counterbalanced across animals. In Sample 1, rats were placed in the arena and were able to move freely around the entire maze and to explore four identical objects. After 5 -min exploration time, the rats were removed from the arena and returned to a holding room. After a 60-min delay, the test phase occurred during which rats were able to explore a pair of objects previously encountered in the sample phase (familiar objects) and two identical novel objects (Fig. 2d). Each end of the maze contained one novel and one familiar object. The test phase lasted $3 \mathrm{~min}$.

\section{Histology}

At the end of the behavioural experiments, the rats were deeply anaesthetised with sodium pentobarbital $(60 \mathrm{mg} / \mathrm{kg}$, Euthatal, Rhone Merieux, UK) and transcardially perfused with 0.1-M phosphate buffer saline (PBS) followed by $4 \%$ paraformaldehyde in $0.1 \mathrm{M}$ PBS (PFA). The brains were removed and post-fixed in PFA for $4 \mathrm{~h}$ and then transferred to $25 \%$ sucrose overnight at room temperature with rotation. Sections were cut at $40 \mu \mathrm{m}$ on a freezing microtome in the coronal plane.

A one-in-four series of sections was mounted onto gelatin-coated slides and stained with cresyl violet, a Nissl stain, for histological assessment. A second series was collected to process for the visualization of calbindin (Arai et al. 1994; Rogers and Résibois 1992). The dense fibrous calbindin stain within the anteroventral thalami nucleus has been attributed to MTT input (Rogers and Résibois 1992); this stain can, therefore, provide a further measure of the completeness of the MTT lesions. The tissue was treated with a blocking buffer containing 3-5\% normal horse serum (S-2000, Vector Laboratories, UK) in 0.1 M PBS and agitated on a stirrer for between $30 \mathrm{~min}$ and $2 \mathrm{~h}$. Sections were subsequently incubated in primary antibody solution (Swant, Switzerland) (1:10,000 dilutions in $0.2 \%$ Triton-X-100 in PBS containing 1\% normal horse serum), for $24 \mathrm{~h}$ at room temperature. The tissue underwent further washes in 0.1 M PBS and, to complete the reaction, the tissue was incubated in a secondary antibody solution (Dylight-594; horse, anti-mouse; 1:200 dilution in 0.2\% Triton-X-100 in $0.1 \mathrm{M}$ PBS containing $1 \%$ normal horse serum) overnight on a shaker table at room temperature. Following an additional series of washes in 0.1 PBS, the tissue sections were mounted on gelatin-subbed slides, allowed to dry for 1-2 days in the dark, and coverslipped using DPX mounting medium (Lamb, UK).

\section{Data analysis}

Exploration of an object was defined as directing the nose at a distance of $<1 \mathrm{~cm}$ to the item and/or touching it with the nose or the paws (including pushing). Sitting on or turning around the item was not included. If the rats spent time chewing, carrying the items in their mouths, and freezing near or above the items (at a distance of $<1 \mathrm{~cm}$ ), these behaviours were also excluded. The videos were scored blind to lesion group assignment.

A discrimination score (D1) and a ratio (D2) were calculated (Ennaceur and Delacour 1988). The recognition score D1 was calculated by subtracting the time spent exploring the older item from the time spent exploring the recent item. When there were multiple trials (between- and within-block recency), the D1 index was summed across trials (cumulative D1). The D2 ratio takes the differential exploration time for the pair of objects (i.e., the D1 score) and then divides it by the total time spent exploring both items. The D2 ratio yields a ratio between -1 and +1 , where a positive score indicates a preference for the least recent (older) item. For the tests of between- and withinblock recency, which involved multiple trials, the D2 ratio was updated after every trial using the summed (updated D2) data (note that the final updated D2 score is, therefore, not equivalent to the mean of each D2 score for every trial).

The D1 score and D2 ratio were also calculated for the standard object recognition trials (Experiment 1a betweenblock recency sample phase, Experiment 1c). The time spent exploring the novel item was subtracted from the time spent exploring the familiar item (i.e., time noveltime familiar). For the D2 ratio, a positive score indicates a preference for the novel item.

Group differences were examined with between subject ANOVAs. To verify whether animals' performance was 
above chance (i.e., zero), the D1 scores and D2 ratios were compared against zero, using a one-sample $t$ test. The alpha level was set at $p<0.05$.

\section{Results}

\section{Histological analysis of the lesions}

The MTT lesions were quantified on the basis of Nisslstained sections and the absence of calbindin staining in the anteroventral thalamic nucleus (Fig. 3a, b). In Experiment 1, 5 of the 15 lesion animals did not have complete bilateral MTT lesions and were consequently removed from all analyses. In Experiment 2, there was evidence of sparing in 7 cases and so these animals were also removed from all analyses. All remaining cases involved discrete bilateral (Experiment 1) or unilateral (Experiment 2) lesions of the MTT, which were sufficiently anterior, so there was no direct damage to the supramammillary nuclei, the mammillary bodies, or the mammillotegmental tract. Similarly, the lesions did not encroach on the postcommissural portion of the fornix (Fig. 3a, b). In Experiment 2, the nine unilateral MTT lesions animals also received a unilateral mPFC lesion in the contralateral hemisphere to the MTT lesion site. The animals exhibited substantial unilateral cell loss within the mPFC. The entire infralimbic cortex was atrophied, with damage also extending into the dorsal peduncular cortex. The prelimbic cortex as well as the rostral anterior cingulate cortex were also absent, except in one case where there was substantial sparing in the dorsal aspect. This animal was, therefore, excluded, leaving 8 cases with crossed MTT-mPFC lesions.

\section{Behavioural results}

\section{Experiment 1a: The effect of MTT lesions on multi-item recency judgments}

Between-block recency The cumulative exploration time during the sample phases did not differ by lesion $(F<1)$. However, analysis of the cumulative D1 score (i.e., the cumulative difference in time spent exploring the novel versus the familiar objects during the sample phases) revealed a main effect of lesion $\left(F_{(1,21)}=12.6, p<0.01\right)$ as well as a trial by lesion interaction $\left(F_{(7,147)}=16.5\right.$, $p<0.001)$, indicating that recognition performance involving continuous trials of standard object recognition with a 1-min delay was impaired in the MTTx relative to the Sham group (Fig. 4). This impairment was not absolute as performance in the MTTx group was above chance (i.e., 0$)$ at the end of each sample phase $\left(\min t_{(9)} 2.8\right.$, $p<0.05)$.

In the test phase, Sham animals successfully discriminated between the objects on the basis of relative recency (i.e., a preferential exploration of objects from Sample 1 compared with Sample 2), whereas the MTTx group showed no preference for objects presented in Sample 1 (Fig. 5a). This difference was reflected by a main effect of lesion $\left(F_{(1,21)} 20.5, p<0.001\right)$. Test performance in Sham $\left(t_{(12)}=5.4, \quad p<0.001\right)$, but not $\operatorname{MTTx}\left(t_{(9)}=-1.2\right.$, $p=0.26$ ) animals was above chance. However, overall exploration time during the test phase did not differ by lesion $(F<1)$.

Within-block recency The cumulative exploration time during the sample phase did not differ by lesion group $(F<1)$. Similarly, there was no effect of lesion on overall levels of exploration during the test phase $(F<1)$.

Test performance was initially analysed by grouping the number of interleaving items into low (3 or 7 interleaving objects) or high (11 or 15 interleaving objects). ANOVA revealed no effect of the number of interleaving items on performance $(F<1)$ or an interaction with lesion group $(F<1)$. Consequently, the data were collapsed across the high and low interleaving items. As is clear from Fig. 5b, the MTTx group was impaired relative to the Sham animals $\left(F_{(1,21)}=7.1, p<0.05\right)$. One-sample $t$ tests confirmed that test performance in Sham animals was above chance $\left(t_{(12)}=3.3, p<0.01\right)$, but performance in the MTTx group did not differ from chance $(t<0)$.

\section{Experiment $1 b$ : the effect of MTT lesions on single-item recency judgments}

Total exploration time during both the sample and test phases did not differ by lesion group $(F<1)$.

In test phase, both groups showed a preference for the item that had been presented in the first temporal block (Fig. 6a) and there was no effect of lesion on test performance $\left(F_{(1,21)}=1.9, p=0.2\right)$. One-sample $t$ tests confirmed that both groups were able to make recency judgements about single items presented in distinct temporal blocks (Sham $t_{(12)}=5.3, p<0.001$; MTT $t_{(9)}=2.7$, $p<0.05)$.

\section{Experiment 1c: the effect of MTT lesions on standard} object recognition

There was a non-significant trend towards overall higher levels of exploration during the sample phase in the MTTx group $\left[\left(F_{(1,21)}=3.6, p=0.07\right.\right.$; Mean total exploration time $\quad( \pm$ S.E.M. $) \quad$ Sham $=59.5 \quad( \pm 3.1) ; \quad$ MTTx $=69.3$ 


\section{A Unilateral MTT lesion}
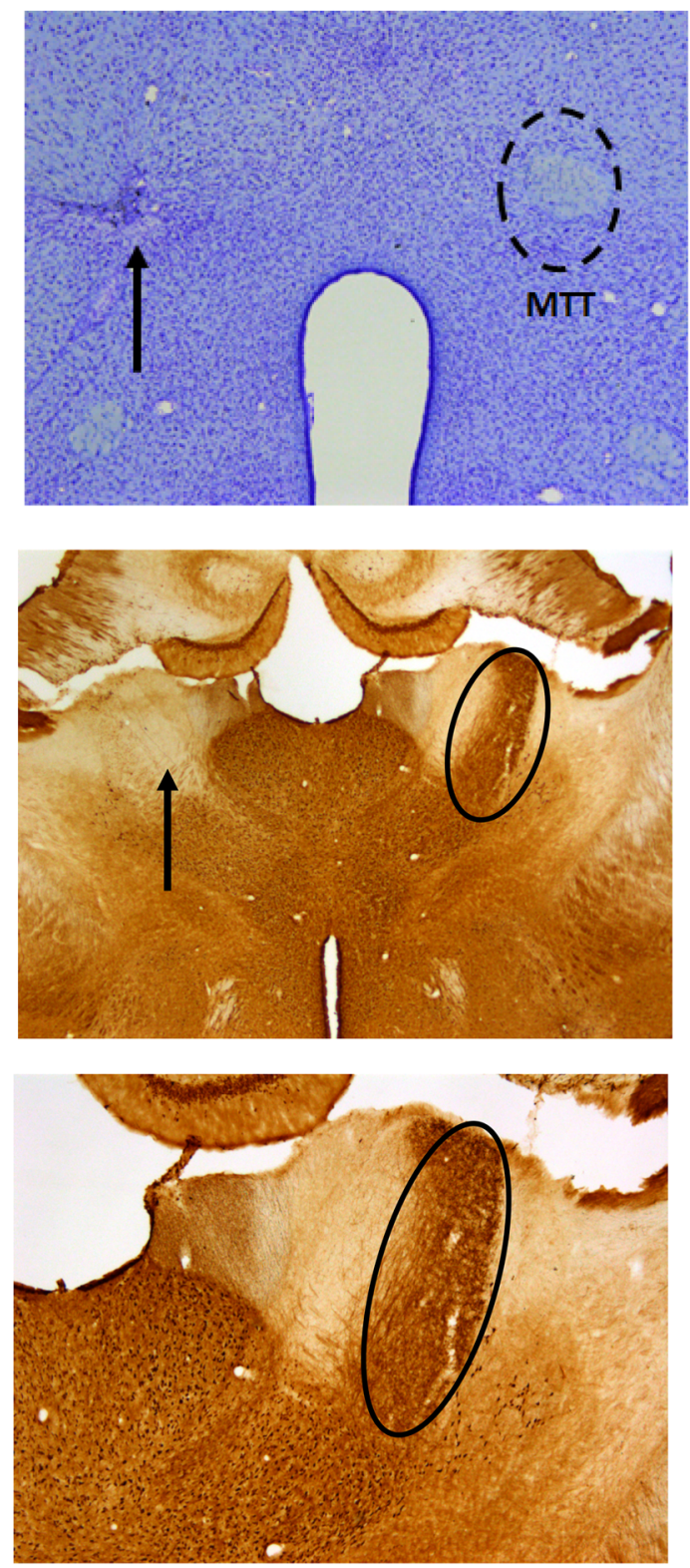

C Unilateral mPFC lesion

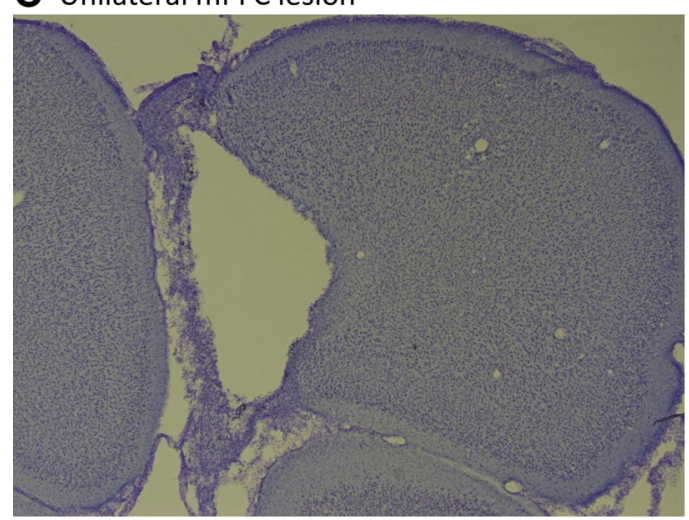

\section{B Bilateral MTT lesion}
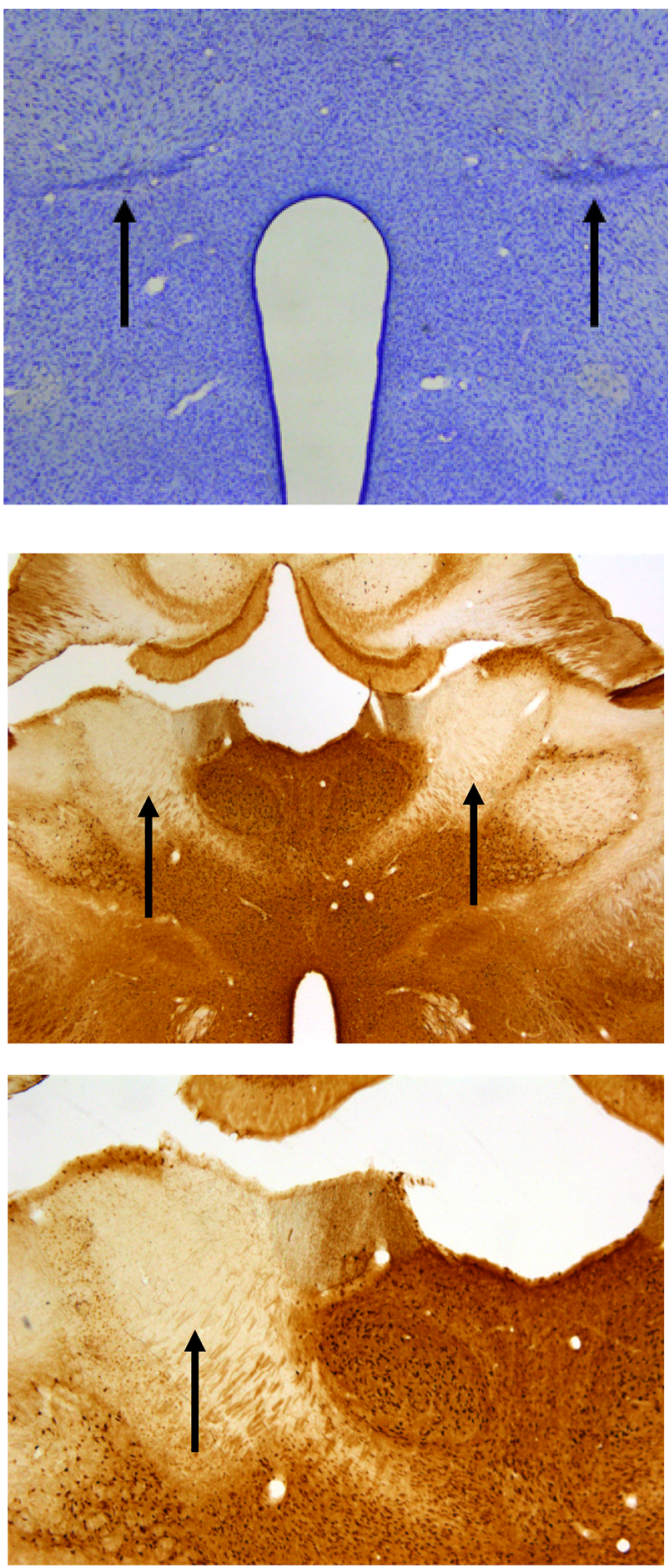

D

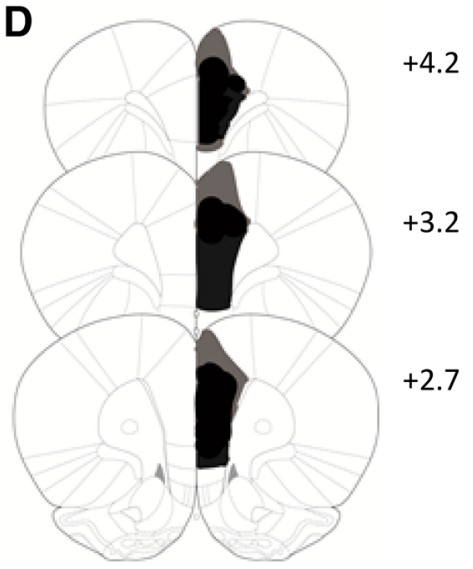


4 Fig. 3 Location and histological verification of mammillothalamic tract (MTT) and medial prefrontal cortex (mPFC) lesions. Photomicrograph of a coronal section immunostained for Nissl (top panel) and for calbindin in the anterior thalamus (middle and bottom panels) showing a unilateral MTT lesion (a) and a bilateral MTT lesion (b). Note the marked loss of calbindin stain in the anteroventral nucleus in the MTT lesion hemispheres. $\mathbf{c}$ Photomicrograph of a coronal section stained for Nissl showing a unilateral mPFC lesion. d Coronal reconstructions showing cases with the minimal (black) extent and the maximal (black and grey areas) extent of the unilateral mPFC lesions. The numbers in (d) indicate the distance (in millimeters) from bregma (adapted from Paxinos and Watson 2005)

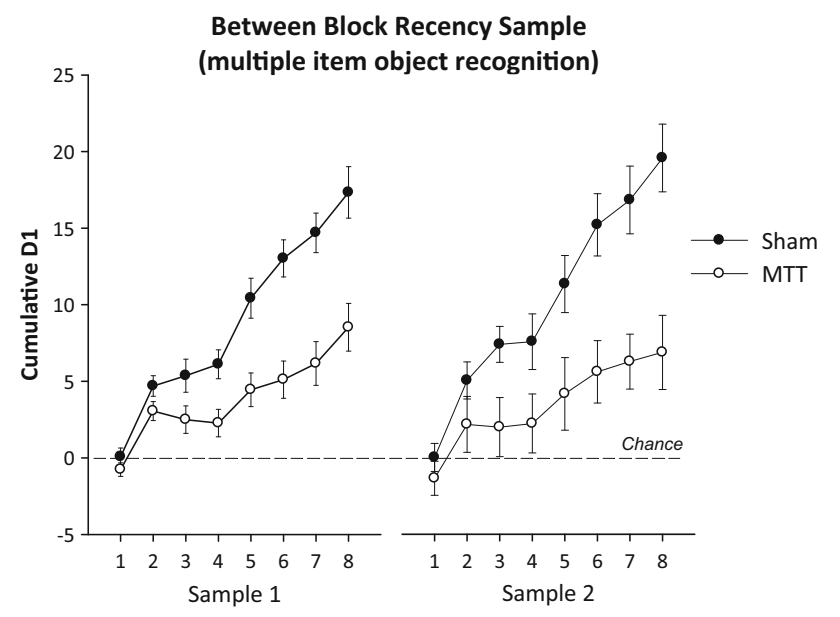

Fig. 4 Cumulative D1 scores of the Sham and MTT groups across the sample phases of the multi-item between-block recency test during which the rats were tested on object recognition with a $1 \mathrm{~min}$ retention interval

$( \pm 4.3)]$. There was a similar trend during the test session $\left[F_{(1,21)}=3.9, \quad p=0.06\right.$; mean total exploration time ( \pm S.E.M.) Sham $=36.6( \pm 2.2) ;$ MTTx $=44.4( \pm 3.5)]$.
Analysis of the D2 ratios from the test phase revealed that both Sham $\left(t_{(12)}=4.1 p<0.01\right)$ and the MTTx animals $\left(t_{(9)}=4.1 p<0.01\right)$ showed a preference for the novel object at test, indicating that both groups were able to discriminate objects in terms of relative familiarity after a 60-min delay (Fig. 6b). Object recognition performance did not differ by group $(F<1)$.

\section{Experiment 2: the effect of crossed MTT-mPFC lesions on multi-item recency judgments}

Between-block recency There was no difference between the groups in the total cumulative exploration time in either sample phase $\left(\max F_{(1,18)}=1.4, p=0.24\right)$. Equally, total exploration time during the test phase was unaffected by lesion $(F<1)$.

At test, both groups showed a preference for items that had been presented least recently (i.e., in Sample 1) (Fig. 7a). Performance in both Sham2 $\left(t_{(11)} 5.9, p<0.001\right)$ and the MTT-mPFC group $\left(t_{(7)}=4.4, p<0.01\right)$ was above chance. There was no effect of lesion on test performance $(F<1)$.

Within-block recency The total cumulative exploration time during the sample and test phases did not differ by lesion group (both $F<1$ ).

The initial analysis revealed no effects of the number of interleaving items on test performance $(F<1)$, and consequently, the test data were collapsed across the number of interleaving items. As is clear from Fig. 7b, both Sham and MTT-mPFC groups were able to discriminate on the basis of relative recency between items presented within the same list and there was no difference between the groups $(F<1)$. One-sample $t$ tests indicated that both groups showed a preference for the least recently presented (older) items (minimum $t_{(7)}=3.3, p<0.05$ ).
Fig. 5 Updated D2 scores for the recency test phases of the between-block (a) and withinblock (b) multi-item recency task. The mean D2 scores of the Sham and MTT groups are shown ( \pm S.E.M.). Hash denotes significantly different from chance, $p<0.05$. Asterisks denotes significant group difference, $p<0.05$

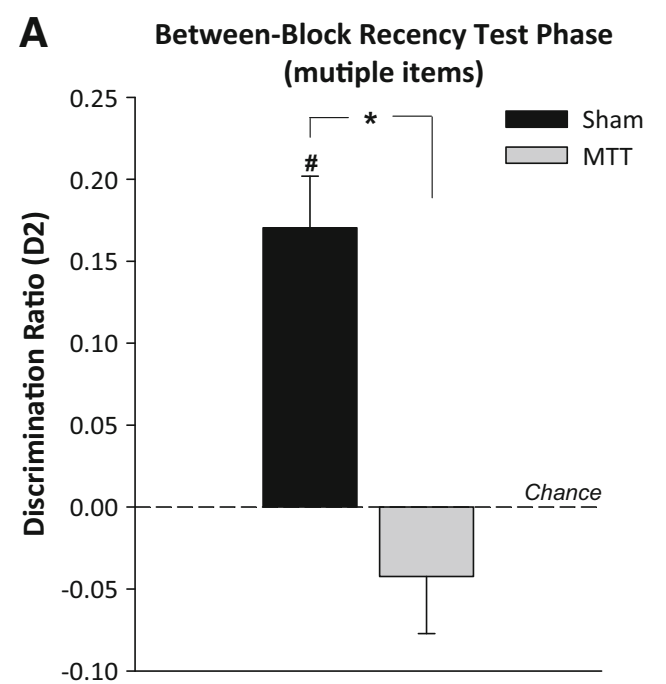

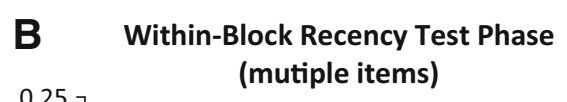

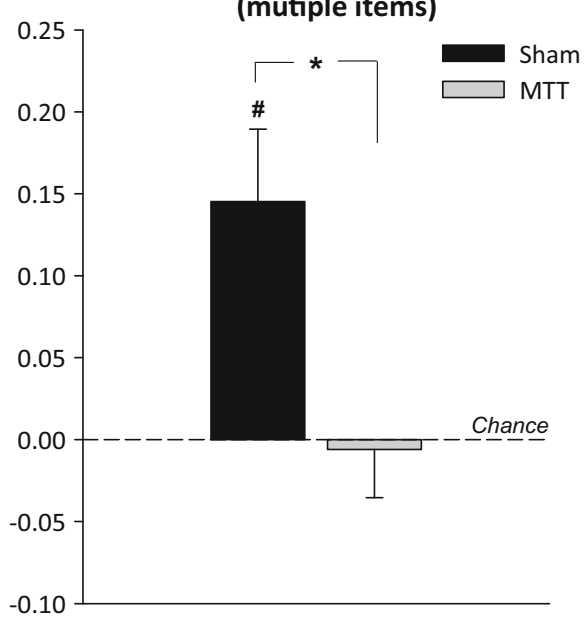


Fig. 6 Mean D2 scores for the simple between-block recency test (a) and test of standard object recognition after a 60-min delay (b). The mean D2 scores of the Sham and MTT groups are shown $( \pm$ S.E.M. $)$. Hash denotes significantly different from chance, $p<0.05$

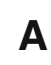

A

Between Block Recency (two items)
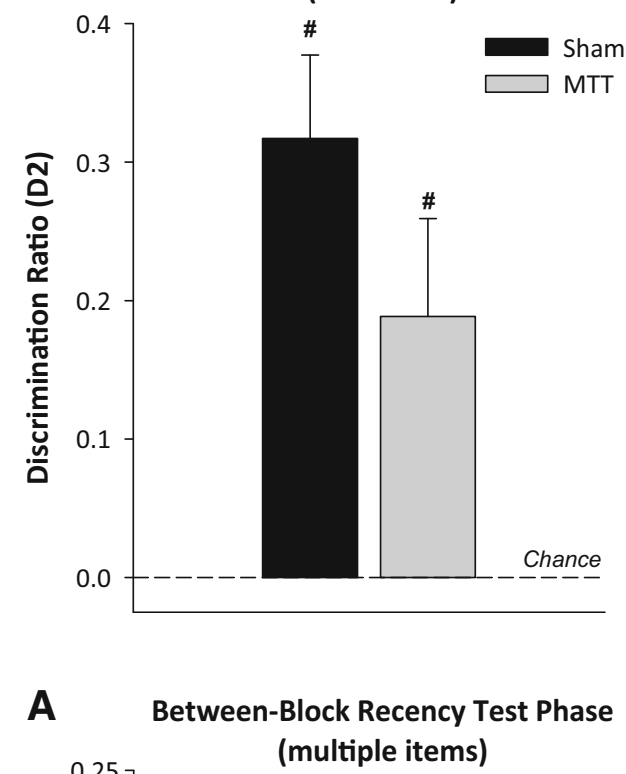

the recency test phases of the between-block (a) and withinblock (b) multi-item recency task. The mean D2 scores of the Sham and crossed MTT-mPFC groups are shown $( \pm$ S.E.M.). Hash denotes significantly different from chance, $p<0.05$

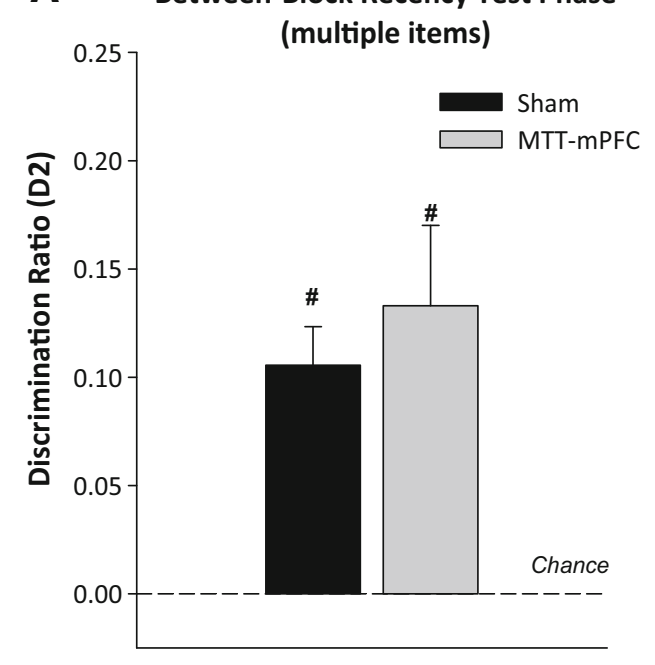

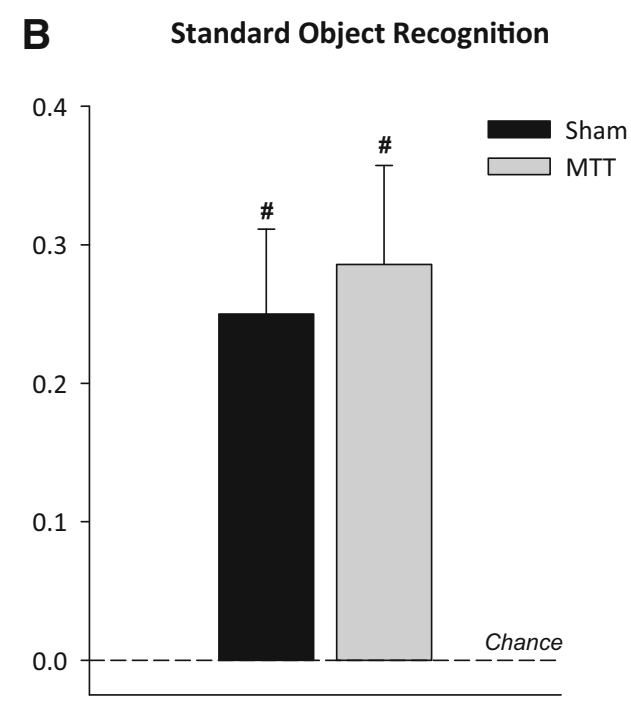

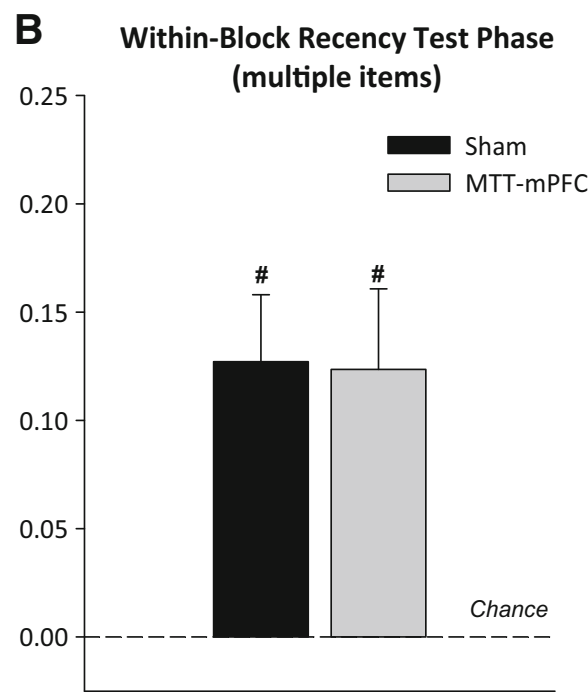

\section{Discussion}

The mammillary bodies were first implicated in memory in the 19th century, but it is still unclear how they support memory (Kapur et al. 1996, 1998; Vann and Nelson 2015; Victor 1987). More broadly, there remains considerable controversy surrounding the core features of diencephalic amnesia as well as the critical site for amnesia to occur (Kopelman 2015). One consistent neuropsychological finding in diencephalic patients is impaired temporal/recency memory; but as the damage in this patient group is anatomically diffuse and often includes pathology beyond the medial diencephalon, a little progress has been made in establishing the precise neuropathology underpinning this deficit. This consideration underscores the importance of comparative lesion studies. Consequently, we examined the impact of selective transection of the rat mammillothalamic tract (MTT) on tests of recency memory analogous to the tasks used in patients (e.g., Hunkin et al. 2015). The distinct advantage of this approach is that it selectively disconnects mammillary body inputs to the anterior thalamic nuclei (Vann et al. 2007), while sparing other thalamic nuclei, such as the mediodorsal thalamus, intralaminar nuclei, and midline thalamic nuclei, that are often affected by the diffuse pathology seen in Korsakoff syndrome (Kopelman 2015; Mitchell and Chakraborty 2013). As such, this experimental approach provides a direct test of the selective importance of mammillary body efferents for recency memory. In line with the deficits observed in Korsakoff's patients (Hunkin et al. 2015), the MTT lesion rats were severely impaired on tests of both 'between' and 'within' block recency memory. These results demonstrate for the first time the selective importance of the mammillary body efferents to the anterior thalamus for recency memory.

On both between- and within-block tests of recency memory, the MTT rats performed at chance level, 
consistently failing to discriminate between multiple objects on the basis of relative recency. An inability to recognise objects over a longer delay is an unlikely account of this pattern of results as the same animals performed at equivalent levels to sham animals on a test of object recognition after a 60-min delay (Fig. 6b). Similarly, when required to discriminate on the basis of relative recency between just two items that had been presented in different time blocks (Fig. 6a), performance in the MTT animals did not differ from sham levels. Even though these control tasks were run after the multiple-item recency tests, the lack of lesion effect is unlikely to reflect training related improvements given that the animals were at no point trained on a rule, i.e., they were not rewarded for choosing the correct (older) item so discrimination performance remained spontaneous throughout. Furthermore, there was no improvement when animals were moved from betweenblock to within-block testing. In contrast to the null effects on the simple object recognition task, a lesion impairment also emerged during the sample phases of the betweenblock task during which the animals were presented with multiple consecutive familiarity discriminations (Fig. 4), a procedure likely to increase stimulus interference. Interestingly, this deficit contrasts with the effect of hippocampal lesions which spare performance on tests of continuous object recognition (Albasser et al. 2012). Nevertheless, this deficit was not absolute as the MTT group's performance remained above chance, i.e., these animals were still able to discriminate between multiple novel and familiar items, albeit less effectively than sham animals.

That impairments only emerged when the animals were required to make familiarity or recency judgements involving multiple objects indicates that impoverished recognition memory or a global deficit in processing temporal information cannot account for the effects of MTT damage on performance. Rather, this profile of deficits points to a specific problem with distinguishing between multiple items or events. One seemingly plausible explanation of this dissociation is that MTT damage leads to heightened sensitivity to the effects of proactive interference. Given that the most profound deficit emerged on the recency trials, it seems, however, unlikely that greater sensitivity to proactive interference can provide a complete account of this deficit, as this factor would presumably affect both familiarity and recency judgments alike. Similarly, the MTT animals were not differentially impaired on the within- relative to the between-block test, despite the latter test potentially reducing interference effects through the additional information provided by the distinct temporal blocks in which stimuli were presented. Moreover, evidence from delayed matching and non-matching to sample procedures provides a little support for the suggestion that mammillary body damage disrupts mnemonic processes through increased susceptibility to proactive interference (Aggleton et al. 1995; Harper et al. 1994; Vann and Aggleton 2003). Instead, the current findings indicate that the mammillary bodies are important for processing associative recognition information (Nelson and Vann 2014) and, in particular, the temporal context in which stimuli are presented. This suggestion accords with the clinical picture. While there have been a few reports of recognition memory deficits and abnormalities in the release from proactive interference in diencephalic amnesic patients (e.g., Kopelman and Stanhope 1998; Squire 1982), profound impairments in recency judgements have been reported in almost all Korsakoff cases (Kopelman 2015), and critically, these deficits have been observed in cases with intact familiarity judgements and normal sensitivity to proactive interference (Hildebrandt et al. 2001; Hunkin and Parkin 1993; Shaw and Aggleton 1995).

This impairment on recency tasks has typically been attributed to co-occurring frontal dysfunction (Mayes et al. 1985; Shimamura et al. 1990; Squire 1982) or disruption to thalamo-frontal circuits (Mair et al. 1979). While there is evidence that disrupting connections between the thalamus and frontal cortex can impair recency memory (Aggleton et al. 2011; Cross et al. 2012; Schnider et al. 1996), the current results demonstrate that restricted damage to the mammillary body-anterior thalamic axis that does not involve frontal associated thalamic nuclei, such as the mediodorsal thalamus, is in itself sufficient to produce marked impairments on tests of recent memory. However, damage to the medial diencephalon, in both rats and patients, can result in functional disruption to frontal cortex. There is very good evidence that diencephalic pathology can produce diaschisis in cortical regions, including frontal cortex (Baron et al. 1992; Fazio et al. 1992; Ozyurt et al. 2014; Paller et al. 1997; Pepin and Auray-Pepin 1993; Reed et al. 2003). Similarly, animal models have shown that selective MTT lesions can cause hypoactivity, as measured by immediate-early gene expression, in the prelimbic cortex (Vann and Albasser 2009; Vann 2013). The mammillary bodies can act indirectly on the prefrontal cortex via their connections with the anteromedial thalamic nucleus, which, in turn, projects to the prefrontal cortex (de Lima et al. 2016; Hoover and Vertes 2007; van Groen et al. 1999). Furthermore, the medial mammillary bodies are innervated directly by the medial prefrontal cortex (Allen and Hopkins 1989). It, therefore, remains possible that the present impairment seen following MTT lesions is driven by the loss of these direct and indirect prefrontal connections or by lesion-induced covert pathology and dysfunction in the prefrontal cortex. To examine the potential functional importance of interactions between the mammillary bodies and the medial 
prefrontal cortex for recency memory, we used disconnection procedures. In stark contrast to the effects of bilateral MTT lesions, the rats with MTT and contrahemispherical medial prefrontal cortex lesions performed at normal levels on both the within- and between-block recency tasks. The implication is that the MTT lesion effects on tests of recency memory seen in Experiment 1 cannot be ascribed to either disconnection of the direct and indirect mammillary body-prefrontal pathway or MTT lesion-induced diaschisis. While there are undoubtedly differences between the human and rodent frontal cortex (Preuss 1995), there are, nevertheless, a number of functional consistencies across species (Uylings et al. 2003). Indeed, recency memory is sensitive to frontal damage in both rodents (e.g., Barker et al. 2007) and primates (e.g., Milner et al. 1991; Petrides 1991), making this an appropriate model to test these functional contributions. While the effects of prefrontal damage have not been tested on the multi-item tests of recency used in this study, it seems reasonable to assume that performance on these tasks would be sensitive to prefrontal damage given that prefrontal lesions consistently disrupt recency memory for single items presented in distinct temporal blocks (Barker et al. 2007; Cross et al. 2012; Hannesson et al. 2004; Nelson et al. 2011).

This study has addressed a long-standing and unresolved issue regarding the neuroanatomical basis of impoverished temporal memory in diencephalic amnesia. Using an animal model, we have shown that damage limited to the MTT can produce marked impairments on tests of recency memory analogous to those used in patients (Hunkin et al. 2015). Furthermore, the results of the disconnection study (Experiment 2) demonstrate that these effects are not due to the loss of interactions with the prefrontal cortex or distal effects of the MTT lesion on the prefrontal cortex. These results reveal for the first time the importance of the medial mammillary body inputs to the anterior thalamus for temporal discriminations. These findings point to the existence of two distinct but presumably complementary mechanisms for recency memory within the medial diencephalon: one involving the mammillary body-anterior thalamus axis (Dumont and Aggleton 2013; Wolff et al. 2006) and the other the mediodorsal thalamus (Aggleton et al. 2011; Cross et al. 2012; Mitchell and Chakraborty 2013; Mitchell and Dalrymple-Alford 2005). These distinct pathways can be dissociated behaviourally. Mediodorsal thalamic lesions, unlike damage to either the MTT or the anterior thalamus, disrupt recency memory for single items presented in distinct temporal blocks (Cross et al. 2012; Dumont and Aggleton 2013; Mitchell and Dalrymple-Alford 2005). Conversely, the role of the mammillary bodyanterior thalamic axis would appear to be restricted to recency judgements involving multiple items (Dumont and
Aggleton 2013). Furthermore, the mediodorsal thalamus and the prefrontal cortex appear to interact functionally to support simple between-block recency judgements (Cross et al. 2012). In contrast, the results from Experiment 2 suggest that the involvement of the mammillary bodies in recency memory does not require interactions with the prefrontal cortex. The presence of these distinct pathways may, in turn, explain why recency memory deficits are particularly prevalent in patient groups with medial diencephalic pathology. On the basis of the current results, it would seem likely that the mammillary bodies are required for fine-grained temporal discriminations when distinguishing between multiple stimuli. One potential role for the mammillary bodies in these processes may be through the regulation of theta, as the majority of cells in the medial mammillary nuclei modulate their firing rate at a frequency of theta (Bland et al. 1995; Dillingham et al. 2015a; Kocsis and Vertes 1994; Vann and Aggleton 2004). It has been suggested that theta provides an oscillatory activity pattern that may help separate temporal events (Dillingham et al. 2015b; Hasselmo and Eichenbaum 2005; Hasselmo and Stern 2014; Nyhus and Curran 2010). Mammillary body or MTT damage might, therefore, disrupt theta oscillations within Papez circuit, resulting in impaired temporal memory.

Acknowledgements The authors wish to thank Moira Davies and Heather Phillips for behavioural testing and histological processing.

\section{Compliance with ethical standards}

Funding This works was funded by a Wellcome Trust Senior Research Fellowship awarded to SDV (Grant Number WT090954AIA).

Conflict of interest The authors declare they have no conflict of interest.

Ethical approval All applicable international, national, and/or institutional guidelines for the care and use of animals were followed. All procedures performed in studies involving animals were in accordance with the ethical standards of the institution or practice at which the studies were conducted.

Open Access This article is distributed under the terms of the Creative Commons Attribution 4.0 International License (http://crea tivecommons.org/licenses/by/4.0/), which permits unrestricted use, distribution, and reproduction in any medium, provided you give appropriate credit to the original author(s) and the source, provide a link to the Creative Commons license, and indicate if changes were made.

\section{References}

Aggleton JP, Neave N, Nagle S, Hunt PR (1995) A comparison of the effects of anterior thalamic, mamillary body and fornix lesions on reinforced spatial alternation. Behav Brain Res 68:91-101. doi:10.1016/0166-4328(94)00163-A 
Aggleton JP, Dumont JR, Warburton EC (2011) Unraveling the contributions of the diencephalon to recognition memory: a review. Learn Mem 18:384-400. doi:10.1101/lm.1884611

Albasser MM, Chapman RJ, Amin E, Iordanova MD, Vann SD, Aggleton JP (2010) New behavioral protocols to extend our knowledge of rodent object recognition memory. Learn Mem 17:407-419. doi:10.1101/lm.1879610

Albasser MM, Amin E, Lin T-CE, Iordanova MD, Aggleton JP (2012) Evidence that the rat hippocampus has contrasting roles in object recognition memory and object recency memory. Behav Neurosci 126:659-669. doi:10.1037/a0029754

Allen GV, Hopkins DA (1989) Mamillary body in the rat: topography and synaptology of projections from the subicular complex, prefrontal cortex, and midbrain tegmentum. J Comp Neurol 286:311-336. doi:10.1002/cne.902860303

Arai R, Jacobowitz DM, Deura S (1994) Distribution of calretinin, calbindin-D28 k, and parvalbumin in the rat thalamus. Brain Res Bull 33:595-614. doi:10.1016/0361-9230(94)90086-8

Barker GRI, Bird F, Alexander V, Warburton EC (2007) Recognition memory for objects, place, and temporal order: a disconnection analysis of the role of the medial prefrontal cortex and perirhinal cortex. J Neurosci 27:2948-2957. doi:10.1523/JNEUROSCI. 5289-06.2007

Baron JC, Levasseur M, Mazoyer B, Legault-Demare F, Mauguière F, Pappata S, Jedynak P, Derome P, Cambier J, Tran-Dinh S (1992) Thalamocortical diaschisis: positron emission tomography in humans. J Neurol Neurosurg Psychiatry 55:935-942

Bland BH, Konopacki J, Kirk IJ, Oddie SD, Dickson CT (1995) Discharge patterns of hippocampal theta-related cells in the caudal diencephalon of the urethan-anesthetized rat. J Neurophysiol 74:322-333

Carlesimo GA, Lombardi MG, Caltagirone C (2011) Vascular thalamic amnesia: a reappraisal. Neuropsychologia 49:777-789. doi:10.1016/j.neuropsychologia.2011.01.026

Cross L, Brown MW, Aggleton JP, Warburton EC (2012) The medial dorsal thalamic nucleus and the medial prefrontal cortex of the rat function together to support associative recognition and recency but not item recognition. Learn Mem 20:41-50. doi:10. 1101/lm.028266.112

de Lima MAX, Baldo MVC, Canteras NS (2016) A role for the anteromedial thalamic nucleus in the acquisition of contextual fear memory to predatory threats. Funct, Brain Struct. doi:10. 1007/s00429-016-1204-2

Devito LM, Eichenbaum H (2011) Memory for the order of events in specific sequences: contributions of the hippocampus and medial prefrontal cortex. J Neurosci 31:3169-3175. doi:10.1523/ JNEUROSCI.4202-10.2011

Dillingham CM, Frizzati A, Nelson AJD, Vann SD (2015a) How do mammillary body inputs contribute to anterior thalamic function? Neurosci Biobehav Rev 54:108-119. doi:10.1016/j.neu biorev.2014.07.025

Dillingham CM, Holmes JD, Wright NF, Erichsen JT, Aggleton JP, Vann SD (2015b) Calcium-binding protein immunoreactivity in Gudden's tegmental nuclei and the hippocampal formation: differential co-localization in neurons projecting to the mammillary bodies. Front Neuroanat 9:1. doi:10.3389/fnana.2015. 00103

Dumont JR, Aggleton JP (2013) Dissociation of recognition and recency memory judgments after anterior thalamic nuclei lesions in rats. Behav Neurosci 127:415-431. doi:10.1037/a0032750

Ennaceur A, Delacour J (1988) A new one-trial test for neurobiological studies of memory in rats. 1: Behavioral data. Behav Brain Res 31:47-59

Fazio F, Perani D, Gilardi MC, Colombo F, Cappa SF, Vallar G, Bettinardi V, Paulesu E, Alberoni M, Bressi S (1992) Metabolic impairment in human amnesia: a PET study of memory networks. J Cereb Blood Flow Metab 12:353-358. doi:10. 1038/jcbfm.1992.52

Gudden H (1896) Klinische und anatomische Beiträge zur Kenntniss der multiplen Alkoholneuritis nebst Bemerkungen über die Regenerationsvorgänge im peripheren Nervensystem. Arch Psychiatr Nervenkr 28:643-741. doi:10.1007/BF01988269

Hannesson DK, Vacca G, Howland JG, Phillips AG (2004) Medial prefrontal cortex is involved in spatial temporal order memory but not spatial recognition memory in tests relying on spontaneous exploration in rats. Behav Brain Res 153:273-285. doi:10. 1016/j.bbr.2003.12.004

Harding A, Halliday G, Caine D, Kril J (2000) Degeneration of anterior thalamic nuclei differentiates alcoholics with amnesia. Brain 123(Pt 1):141-154

Harper C (2009) The neuropathology of alcohol-related brain damage. Alcohol Alcohol 44:136-140. doi:10.1093/alcalc/ agn 102

Harper C, Corbett D (1990) Changes in the basal dendrites of cortical pyramidal cells from alcoholic patients-a quantitative Golgi study. J Neurol Neurosurg Psychiatry 53:856-861

Harper DN, McLean AP, Dalrymple-Alford JC (1994) Forgetting in rats following medial septum or mammillary body damage. Behav Neurosci 108:691-702

Hasselmo ME, Eichenbaum H (2005) Hippocampal mechanisms for the context-dependent retrieval of episodes. Neural Netw 18:1172-1190. doi:10.1016/j.neunet.2005.08.007

Hasselmo ME, Stern CE (2014) Theta rhythm and the encoding and retrieval of space and time. Neuroimage 85(Pt 2):656-666. doi:10.1016/j.neuroimage.2013.06.022

Hayakawa T, Zyo K (1989) Retrograde double-labeling study of the mammillothalamic and the mammillotegmental projections in the rat. J Comp Neurol 284:1-11. doi:10.1002/cne.902840102

Hildebrandt H, Müller S, Bussmann-Mork B, Goebel S, Eilers N (2001) Are some memory deficits unique to lesions of the mammillary bodies? J Clin Exp Neuropsychol 23:490-501. doi:10.1076/jcen.23.4.490.1234

Hoover WB, Vertes RP (2007) Anatomical analysis of afferent projections to the medial prefrontal cortex in the rat. Brain Struct Funct 212:149-179. doi:10.1007/s00429-007-0150-4

Hunkin NM, Parkin AJ (1993) Recency judgements in WernickeKorsakoff and post-encephalitic amnesia: influences of proactive interference and retention interval. Cortex 29:485-499

Hunkin NM, Awad M, Mayes AR (2015) Memory for between-list and within-list information in amnesic patients with temporal lobe and diencephalic lesions. J Neuropsychol 9:137-156. doi:10.1111/jnp.12040

Huppert FA, Piercy M (1976) Recognition memory in amnesic patients: effect of temporal context and familiarity of material. Cortex 12:3-20

Kapur N, Thompson S, Cook P, Lang D, Brice J (1996) Anterograde but not retrograde memory loss following combined mammillary body and medial thalamic lesions. Neuropsychologia 34:1-8

Kapur N, Crewes H, Wise R, Abbott P, Carter M, Millar J, Lang D (1998) Mammillary body damage results in memory impairment but not amnesia. Neurocase 4:509-517. doi:10.1080/ 13554799808410643

Kocsis B, Vertes RP (1994) Characterization of neurons of the supramammillary nucleus and mammillary body that discharge rhythmically with the hippocampal theta rhythm in the rat. J Neurosci 14:7040-7052

Kopelman MD (1989) Remote and autobiographical memory, temporal context memory and frontal atrophy in Korsakoff and Alzheimer patients. Neuropsychologia 27:437-460. doi:10.1016/ 0028-3932(89)90050-X

Kopelman MD (2015) What does a comparison of the alcoholic Korsakoff syndrome and thalamic infarction tell us about 
thalamic amnesia? Neurosci Biobehav Rev 54:46-56. doi:10. 1016/j.neubiorev.2014.08.014

Kopelman MD, Stanhope N (1998) Recall and recognition memory in patients with focal frontal, temporal lobe and diencephalic lesions. Neuropsychologia 36:785-795

Kopelman MD, Stanhope N, Kingsley D (1997) Temporal and spatial context memory in patients with focal frontal, temporal lobe, and diencephalic lesions. Neuropsychologia 35:1533-1545

Langlais PJ, Zhang SX, Savage LM (1996) Neuropathology of thiamine deficiency: an update on the comparative analysis of human disorders and experimental models. Metab Brain Dis 11:19-37

Mair WG, Warrington EK, Weiskrantz L (1979) Memory disorder in Korsakoff's psychosis: a neuropathological and neuropsychological investigation of two cases. Brain 102:749-783

Mayes AR, Meudell PR, Pickering A (1985) Is organic amnesia caused by a selective deficit in remembering contextual information? Cortex 21:167-202

Mayes AR, Meudell PR, Mann D, Pickering A (1988) Location of lesions in Korsakoff's syndrome: neuropsychological and neuropathological data on two patients. Cortex 24:367-388

McAndrews MP, Milner B (1991) The frontal cortex and memory for temporal order. Neuropsychologia 29:849-859. doi:10.1016/ 0028-3932(91)90051-9

Meudell PR, Mayes AR, Ostergaard A, Pickering A (1985) Regency and frequency judgements in alcoholic amnesics and normal people with poor memory. Cortex 21:487-511

Milner B, Corsi P, Leonard G (1991) Frontal-lobe contribution to recency judgements. Neuropsychologia 29:601-618. doi:10. 1016/0028-3932(91)90013-X

Mitchell AS, Chakraborty S (2013) What does the mediodorsal thalamus do? Front Syst Neurosci 7:37. doi:10.3389/fnsys.2013. 00037

Mitchell AS, Dalrymple-Alford JC (2005) Dissociable memory effects after medial thalamus lesions in the rat. Eur J Neurosci 22:973-985. doi:10.1111/j.1460-9568.2005.04199.x

Nelson AJD, Vann SD (2014) Mammilliothalamic tract lesions disrupt tests of visuo-spatial memory. Behav Neurosci 128:494-503. doi:10.1037/bne0000001

Nelson AJD, Cooper MT, Thur KE, Marsden CA, Cassaday HJ (2011) The effect of catecholaminergic depletion within the prelimbic and infralimbic medial prefrontal cortex on recognition memory for recency, location, and objects. Behav Neurosci 125:396-403. doi:10.1037/a0023337

Nyhus E, Curran T (2010) Functional role of gamma and theta oscillations in episodic memory. Neurosci Biobehav Rev 34:1023-1035. doi:10.1016/j.neubiorev.2009.12.014

Ozyurt J, Lorenzen A, Gebhardt U, Warmuth-Metz M, Müller HL, Thiel CM (2014) Remote effects of hypothalamic lesions in the prefrontal cortex of craniopharygioma patients. Neurobiol Learn Mem 111:71-80. doi:10.1016/j.nlm.2014.03.007

Paller KA, Acharya A, Richardson BC, Plaisant O, Shimamura AP, Reed BR, Jagust WJ (1997) Functional neuroimaging of cortical dysfunction in alcoholic Korsakoff's syndrome. J Cogn Neurosci 9:277-293. doi:10.1162/jocn.1997.9.2.277

Parkin AJ, Leng NR, Hunkin NM (1990) Differential sensitivity to context in diencephalic and temporal lobe amnesia. Cortex $26: 373-380$

Paxinos G, Watson C (2005) The rat brain in stereotaxic coordinates. Elsevier Academic Press, Amsterdam

Pepin EP, Auray-Pepin L (1993) Selective dorsolateral frontal lobe dysfunction associated with diencephalic amnesia. Neurology 43:733-741

Petrides M (1991) Functional specialization within the dorsolateral frontal cortex for serial order memory. Proc Biol Sci 246:299-306. doi:10.1098/rspb.1991.0158
Pitel A-L, Chételat G, Le Berre AP, Desgranges B, Eustache F, Beaunieux H (2012) Macrostructural abnormalities in Korsakoff syndrome compared with uncomplicated alcoholism. Neurology 78:1330-1333. doi:10.1212/WNL.0b013e318251834e

Preuss TM (1995) Do rats have prefrontal cortex? The rose-woolseyakert program reconsidered. J Cogn Neurosci 7:1-24. doi:10. 1162/jocn.1995.7.1.1

Reed LJ, Lasserson D, Marsden P, Stanhope N, Stevens T, Bello F, Kingsley D, Colchester A, Kopelman MD (2003) FDG-PET findings in the Wernicke-Korsakoff syndrome. Cortex 39:1027-1045

Rogers JH, Résibois A (1992) Calretinin and calbindin-D28 k in rat brain: patterns of partial co-localization. Neuroscience 51:843-865

Schnider A, Gutbrod K, Hess CW, Schroth G (1996) Memory without context: amnesia with confabulations after infarction of the right capsular genu. J Neurol Neurosurg Psychiatry 61:186-193

Shaw C, Aggleton JP (1995) Evidence for the independence of recognition and recency memory in amnesic subjects. Cortex 31:57-71

Shimamura AP, Janowsky JS, Squire LR (1990) Memory for the temporal order of events in patients with frontal lobe lesions and amnesic patients. Neuropsychologia 28:803-813

Squire LR (1982) Comparisons between forms of amnesia: some deficits are unique to Korsakoff's syndrome. J Exp Psychol Learn Mem Cogn 8:560-571

Torvik A, Lindboe CF, Rogde S (1982) Brain lesions in alcoholics: a neuropathological study with clinical correlations. J Neurol Sci $56: 233-248$

Uylings HBM, Groenewegen HJ, Kolb B (2003) Do rats have a prefrontal cortex? Behav Brain Res 146:3-17

Van der Werf YD, Scheltens P, Lindeboom J, Witter MP, Uylings HBM, Jolles J (2003) Deficits of memory, executive functioning and attention following infarction in the thalamus; a study of 22 cases with localised lesions. Neuropsychologia 41:1330-1344

Van Groen T, Kadish I, Wyss JM (1999) Efferent connections of the anteromedial nucleus of the thalamus of the rat. Brain Res Rev 30:1-26

Vann SD (2013) Dismantling the Papez circuit for memory in rats. Elife 2:e00736. doi:10.7554/eLife.00736

Vann SD, Aggleton JP (2003) Evidence of a spatial encoding deficit in rats with lesions of the mammillary bodies or mammillothalamic tract. J Neurosci 23:3506-3514. doi:10.23/8/3506

Vann SD, Aggleton JP (2004) The mammillary bodies: two memory systems in one? Nat Rev Neurosci 5:35-44. doi:10.1038/ nrn1299

Vann SD, Albasser MM (2009) Hippocampal, retrosplenial, and prefrontal hypoactivity in a model of diencephalic amnesia: Evidence towards an interdependent subcortical-cortical memory network. Hippocampus 19:1090-1102. doi:10.1002/hipo.20574

Vann SD, Nelson AJD (2015) The mammillary bodies and memory: more than a hippocampal relay. Prog Brain Res 219:163-185. doi:10.1016/bs.pbr.2015.03.006

Vann SD, Saunders RC, Aggleton JP (2007) Distinct, parallel pathways link the medial mammillary bodies to the anterior thalamus in macaque monkeys. Eur J Neurosci 26:1575-1586. doi:10.1111/j.1460-9568.2007.05773.x

Victor M (1987) The irrelevance of mammillary body lesions in the causation of the Korsakoff amnesic state. Int $\mathbf{J}$ Neurol 21-22:51-57

Wolff M, Gibb SJ, Dalrymple-Alford JC (2006) Beyond spatial memory: the anterior thalamus and memory for the temporal order of a sequence of odor cues. J Neurosci 26:2907-2913. doi:10.1523/JNEUROSCI.5481-05.2006

Wright NF, Vann SD, Erichsen JT, O'Mara SM, Aggleton JP (2013) Segregation of parallel inputs to the anteromedial and anteroventral thalamic nuclei of the rat. J Comp Neurol 521:2966-2986. doi:10.1002/cne.23325 